\title{
Prevotella histicola
}

National Cancer Institute

\section{Source}

National Cancer Institute. Prevotella histicola. NCI Thesaurus. Code C128545.

A species of obligately anaerobic, non-motile, Gram-negative bacillus-shaped bacteria in the phylum Bacteroidetes. This species is considered a normal part of the human oral microbiota. P. histicola is considered to be commensal. 